\title{
Gradientes de diversidade nas comunidades de peixes da bacia do rio Iguatemi, Mato Grosso do Sul, Brasil
}

\author{
Yzel Rondon Súarez ${ }^{1} \&$ Miguel Petrere Júnior ${ }^{2}$
}

1. Universidade Estadual de Mato Grosso do Sul, GASLAB, Laboratório de Ecologia, Rodovia Dourados-Itahum, km 12, 79804-970 Dourados, MS. (yzel@uems.br)

2. Universidade Estadual Paulista "Júlio de Mesquita Filho”, Depto de Ecologia, Av. 24A 1515, 13506-900 Rio Claro, SP. (mpetrere@rc.unesp.br)

\begin{abstract}
Diversity gradients in fish communities of Iguatemi river basin, Mato Grosso do Sul, Brazil. In order to verify the importance of longitudinal and seasonal variations and the effect of physical and chemical water characteristics to fish species diversity we accomplished three-month samplings, between November/1999 and August/2000, in three selected points in the Jogui River and eight points in the Iguatemi River-MS. The longitudinal variation was more important than seasonal variation in determining richness and number of individuals in the Jogui River, however, evenness did not present statistically significant differences either longitudinal or seasonal. In the Jogui River $78.3 \%$ of species richness, $81.6 \%$ of number of individuals and $41.1 \%$ of evenness variation was explained by the water chemical and physical characteristics. In the Iguatemi River we did not detect longitudinal or seasonal statistically significant differences in the analyzed community descriptors nor significant influence of environmental factors.
\end{abstract}

KEYWORDS. Community ecology, fish, diversity, Iguatemi river.

RESUMO. Com o objetivo de verificar a importância da variação longitudinal, sazonal e a influência das características físicas e químicas da água sobre a diversidade de espécies de peixes realizamos amostragens trimestrais, entre Novembro/1999 e Agosto/2000, em três pontos amostrais no rio Jogui e oito pontos amostrais no rio Iguatemi-MS. A variação longitudinal foi mais importante que a variação sazonal na determinação da riqueza e número de indivíduos no rio Jogui, no entanto, a equitabilidade não apresentou diferença significativa sazonal ou longitudinal. No rio Jogui $78,3 \%$ da variação na riqueza de espécies, $81,6 \%$ no número de indivíduos e $41,1 \%$ na equitabilidade foram explicadas pelas características físicas e químicas da água. No rio Iguatemi nós não detectamos diferenças estatisticamente significativas longitudinais ou sazonais nos descritores analisados das comunidades nem influência significativa dos fatores ambientais.

PALAVRAS-CHAVE. Ecologia de comunidades, peixes, diversidade, rio Iguatemi.

Dentre as hipóteses utilizadas para explicar a diversidade de espécies nas comunidades biológicas, LOREAu \& MouQuet (1999) destacam dois grupos: 1) as que enfocam o papel dos fatores abióticos e das interações biológicas na organização das comunidades (variação espaço-temporal, competição, predação, mutualismo), onde alguns dos estudos clássicos são os de Pianka (1966), MaCARTHUR \& LeVins (1967) e SCHOENER (1974), quanto à diferenciação de nicho e competição interespecífica e 2) as que enfocam o efeito dos processos em macroescala determinando a colonização de um novo ambiente e as taxas de extinção das populações, sendo o principal trabalho o de MACARTHUR \& WiLSON (1967) sobre a teoria de biogeografia de ilhas, sendo que as conclusões sobre a maior importância das variáveis bióticas ou abióticas são fortemente influenciadas pela escala espacial em que os trabalhos são realizados (JACKSON et al., 2001).

Analisando comunidades lóticas, o conceito de rio contínuo (VANNOTE et al., 1980) estabelece que as características como diversidade, produtividade, relações bióticas, entre outras, se alteram de forma previsível ao longo do curso de um rio. No entanto, existe certa controvérsia quanto à variação na diversidade de espécies em suas diferentes formas (riqueza e equitabilidade, por exemplo) ao longo do gradiente lótico, sendo que alguns estudos sugerem que ocorra a adição de espécies ao longo deste, influenciada pelo aumento da diversidade de microhabitats disponíveis (GARUtTI, 1988; REASH \& PigG, 1990; BENNEMANn et al., 1995; RATHERT et al., 1999; Vila-Gispert et al. 2002; Bistoni \& Hued, 2002). No entanto, Statzner \& Higler (1986), estudando comunidades de invertebrados bentônicos, sugerem que os processos regionais sejam os principais determinantes da organização das comunidades em rios, ocorrendo basicamente a substituição das espécies ao longo do gradiente lótico. Por outro lado, JunK et al. (1989) sugerem que os aspectos hidrológicos juntamente com os aspectos geomorfológicos, produzindo picos de inundações e secas, com diferentes amplitudes e períodos ao longo da bacia hidrográfica, sejam a maior força controladora da biota nos rios com planície de inundação.

Considerando a controvérsia sobre a importância das diferentes fontes de variação nas comunidades, bem como a escassa literatura comparando as diferentes hipóteses em comunidades de peixes neotropicais, o objetivo do presente estudo é responder às seguintes perguntas: 1) Qual fonte de variação (longitudinal vs sazonal) é mais importante na determinação da diversidade de espécies de peixes na bacia do rio Iguatemi? 2) Como as características físicas e químicas dos locais influenciam a diversidade da ictiofauna? 


\section{MATERIAL E MÉTODOS}

O rio Iguatemi, localizado no extremo sul do estado do Mato Grosso do Sul, caracteriza-se como um rio de planície, com curso meandrante e extensas áreas alagáveis. Possui cerca de $300 \mathrm{~km}$ de extensão, nascendo aproximadamente a cerca de $520 \mathrm{~m}$ de altitude. A vegetação típica da região é o cerrado, alternando-se com floresta estacional semidecidual. O rio Iguatemi deságua no rio Paraná a $226 \mathrm{~m}$ de altitude, cerca de $20 \mathrm{~km}$ acima das cachoeiras de Sete Quedas, importante barreira biogeográfica entre o alto e médio rio Paraná, hoje eliminada pela represa de Itaipu.

A agricultura e a pecuária são as principais atividades econômicas da região. No passado houve intensa exploração de madeira (ainda existente principalmente no lado paraguaio) o que, juntamente com a agropecuária, levou ao desmatamento de boa parte da bacia. O desmatamento, interagindo com o relevo acidentado, acelerou o processo de assoreamento de seus afluentes.

Neste estudo foram realizadas quatro amostragens (novembro/1999, fevereiro/2000, maio/2000 e agosto/ $2000)$ com oito redes de espera $(10 \times 1,5 \mathrm{~m})$ com malhas de 20, 30, 40, 50, 60, 70, 80 e 110 mm entre nós adjacentes. As redes foram armadas à tarde e recolhidas na manhã seguinte.

Os locais de amostragem, oito pontos no rio Iguatemi e três no rio Jogui (Fig. 1), foram definidos de acordo com a posição na bacia hidrográfica, aliado à facilidade de deslocamento por terra.

Os locais amostrados, no sentido nascente-trecho final, apresentam uma redução da velocidade média da água e um aumento da turbidez e do volume. O trecho médio do rio Jogui localiza-se entre dois trechos de corredeira, enquanto o trecho final apresenta uma planície inundável em sua margem direita, sendo um dos trechos mais assoreados da bacia, com vários bancos de areia se formando no período da seca. Nos seus trechos intermediários (Iguat-3 a Iguat-5) o rio Iguatemi apresenta-se bem encaixado, praticamente sem áreas inundáveis e com barranco relativamente alto (aproximadamente 2,5 m); em vários trechos ocorrem pequenas corredeiras, com afloramento de rochas que impedem que o rio seja totalmente navegável. Praticamente inexistem macrófitas aquáticas ao longo de ambos os rios, sendo que no trecho final do rio Iguatemi (o mais assoreado), apenas algumas gramíneas ocorrem associadas à margem.

Os peixes foram fixados em formol a $10 \%$ e preservados em etanol a 70\%, para posterior identificação e biometria. Exemplares testemunho encontram-se disponíveis no laboratório de Biologia da UEMS/Unidade de Ivinhema e no Museu de Zoologia da USP (MZUSP).

A influência da variação sazonal, longitudinal e das variáveis ambientais sobre a diversidade nas comunidades foi quantificada para três parâmetros das comunidades: 1) riqueza de espécies, definida como o número de espécies em cada amostra/local; 2) equitabilidade definida como H'/ Hmax, onde H' é a diversidade de Shannon para a amostra e Hmax é a diversidade máxima que esta comunidade alcançaria se todas as espécies tivessem a mesma abundância, ou seja, $\left(\log _{2}(\mathrm{~S})\right)$, sendo $\mathrm{S}$ o número de espécies na amostra e 3 ) número de indivíduos coletados. Tanto a diversidade (H') quanto a diversidade máxima (Hmax) foram obtidas utilizando o $\log _{2}$.

As comparações das médias de riqueza, equitabilidade e número de indivíduos entre os meses (variação sazonal) e ao longo do gradiente lótico (variação longitudinal) foram realizadas através de análises de variância (ANOVA) unifatorial tendo mês e posição longitudinal como fatores. Não foi possível a realização de uma análise bifatorial devido à impossibilidade de amostragem em três locais no mês de

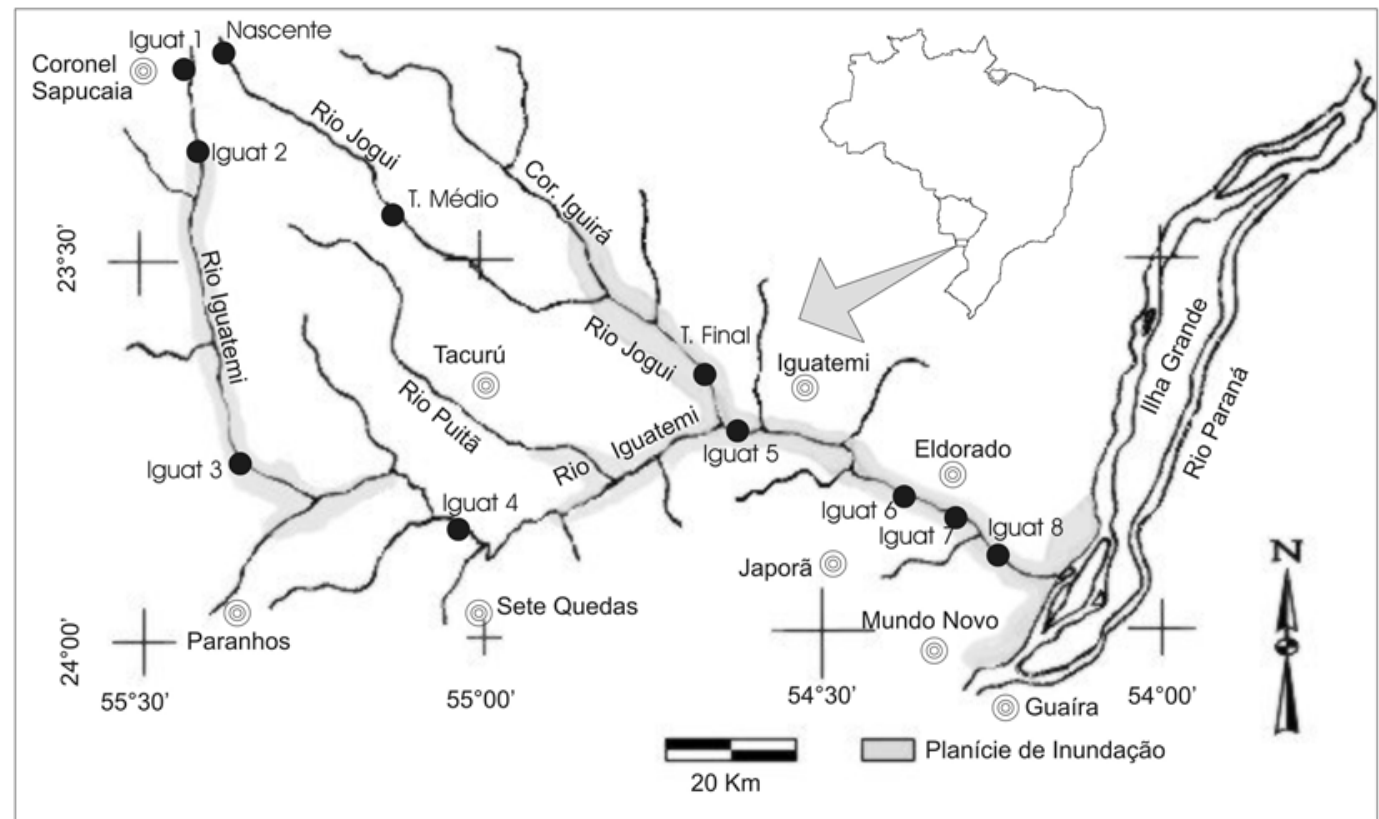

Fig. 1. Mapa da bacia do rio Iguatemi, com os locais amostrados nos rios Jogui e Iguatemi, MS. Pontos amostrais, (0) Municípios na bacia. 
fevereiro (Iguat-4, Iguat-7 e Iguat-8) e pela ausência de captura em outros locais (maio: Iguat-7 e trecho médio do rio Jogui; agosto: Iguat-4 e trecho médio do rio Jogui), o que impede a análise da interação dos fatores. Os resultados da ANOVA foram considerados satisfatórios quando o poder do teste foi igual ou superior a $80 \%$. Quando, por problemas de normalidade ou homocedasticidade (mesmo após transformação), não foi possível a realização da ANOVA, esta foi substituída pelo teste não-paramétrico correspondente (Kruskall-Wallis).

Com o objetivo de quantificar o efeito das características ambientais sobre os descritores das comunidades (riqueza, equitabilidade e número de indivíduos coletados) realizamos análises de regressão linear múltipla stepwise (backward), tendo como variáveis resposta a riqueza, equitabilidade e número de indivíduos e como variáveis explanatórias o $\mathrm{pH}$, condutividade, concentração de oxigênio, turbidez, temperatura da água, velocidade da água e altitude. Com o objetivo de atender aos pressupostos de normalidade e homogeneidade de variâncias, necessários para a Análise de Regressão, os dados foram transformados em $\log _{2}+0,1$. No rio Iguatemi não foi necessária a transformação dos dados de equitabilidade.

A opção pela utilização da riqueza e equitabilidade, ao invés da diversidade de espécies, foi tomada pelo fato da desta última considerar as duas primeiras, dificultando então sua interpretação estatística ou biológica (GOTELLI \& ENTSMINGER, 2001).

\section{RESULTADOS}

Constatamos um aumento na riqueza média ao longo do gradiente longitudinal no rio Jogui (Tab. I, Fig. 2), porém para o rio Iguatemi não é possível visualizar uma variação clara na riqueza ao longo do rio (Tab. I, Fig. 3). No rio Jogui, a riqueza e o número de indivíduos coletados variaram significativamente entre os pontos amostrados, mas não variaram ao longo dos meses (Tab.
II). No entanto, para o rio Iguatemi, nenhum dos parâmetros das comunidades variou significativamente entre as estações do ano e ao longo do gradiente lótico (Tab. III).

Para o rio Jogui, verificamos um aumento da riqueza e equitabilidade ao longo do gradiente lótico. No entanto, o trecho médio sempre apresentou menores valores dos parâmetros analisados (Tab. I, Fig. 2), o que em parte é resultado da ausência de capturas nas amostragens de maio e agosto. Porém, constatamos que não existe uma diferenciação clara entre as amostragens realizadas nas diferentes estações do ano.

A riqueza de espécies no rio Jogui foi significativamente correlacionada com as seguintes variáveis: $\mathrm{pH}$, oxigênio dissolvido, temperatura, turbidez e altitude, em ordem decrescente de importância $\left(r^{2}=0,783\right.$; $\mathrm{F}=4,335 ; \mathrm{N}=12 ; \mathrm{P}=0,05)$, sendo que estas variáveis permitiram explicar $78,3 \%$ da variação na riqueza. A regressão entre o número de indivíduos e as características ambientais permitiu explicar $81,6 \%$ da variação nos dados $\left(r^{2}=0,816 ; \mathrm{F}=7,782 ; \mathrm{N}=12 ; \mathrm{P}=0,01\right)$, sendo que as variáveis mais importantes foram oxigênio dissolvido, condutividade, turbidez e altitude. Não constatamos influência significativa das características ambientais sobre a equitabilidade, sendo que somente $41,1 \%$ da variação nos dados foi explicada pelo modelo $\left(\mathrm{r}^{2}=0,411 ; \mathrm{F}=3,137 ; \mathrm{N}=12 ; \mathrm{P}=0,093\right)$ (Tab. IV).

$\mathrm{O}$ resultado da análise de regressão stepwise entre a riqueza de espécies e as características limnológicas do rio Iguatemi permitiu concluir que nenhuma das variáveis utilizadas é um preditor significativo para a riqueza de espécies $\left(r^{2}=0,289 ; \mathrm{F}=2,442 ; \mathrm{N}=29 ; \mathrm{P}=0,074\right)$. As variáveis condutividade e oxigênio dissolvido influenciam significativamente o número de indivíduos coletados, apresentando correlação positiva com este $\left(r^{2}=0,378\right.$; $\mathrm{F}=7,901 ; \mathrm{N}=29 ; \mathrm{P}=0,002)$. No entanto, os valores parciais de $\mathrm{p}$ mostram que somente a condutividade apresenta influência significativa. A equitabilidade não foi estatisticamente influenciada pelas características dos locais estudados $\left(r^{2}=0,231 ; \mathrm{F}=2,504 ; \mathrm{N}=29 ; \mathrm{P}=0,082\right)$ (Tab. IV).

Tabela I. Valores médios, mínimo e máximo para os descritores das comunidades de peixes e as características ambientais dos locais amostrados, no período de novembro/1999 a agosto/2000 (NInd., Número de indivíduos; Equitab., Equitabilidade; Condut., Condutividade elétrica $\left(\mu \mathrm{S} / \mathrm{cm}^{-1}\right)$; OD, oxigênio dissolvido (mg/l); Temp., Temperatura da água; Veloc., Velocidade da água (m/s)).

\begin{tabular}{|c|c|c|c|c|c|c|c|c|c|c|}
\hline \multicolumn{11}{|c|}{ Rio Jogui } \\
\hline Locais & Riqueza & NInd. & Equitab. & $\mathrm{pH}$ & Condut. & OD & Turbidez & Temp. & Veloc. & Altitude \\
\hline Nascente & $4,5(3-6)$ & $17,3(11-21)$ & $0,93(0,82-0,99)$ & $6,1(5,9-6,3)$ & $23,3(19-30)$ & $8,0(6,6-9,4)$ & $11(3-28)$ & $20,7(19,7-22,0)$ & $0,35(0,30-0,40)$ & 468 \\
\hline T-Médio & $2(1-3)$ & $2(0-6)$ & $0,46(0-0,92)$ & $6,4(6,2-6,8)$ & $19,3(18-20)$ & $7,7(6,3-9,0)$ & $33,3(24-48)$ & $23,0(20,5-25,0)$ & $0,52(0,33-0,83)$ & 324 \\
\hline T-Final & $7,8(7-12)$ & $12,5(9-15)$ & $0,89(0,81-0,97)$ & $6,3(5,5-7,1)$ & $8,3(7-10)$ & $7,3(6,3-9,2)$ & $113,5(61-162)$ & $22,9(18,9-28)$ & $0,28(0,10-0,45)$ & 250 \\
\hline \multicolumn{11}{|c|}{ Rio Iguatemi } \\
\hline Locais & Riqueza & NInd. & Equitab. & $\mathrm{pH}$ & Condut. & OD & Turbidez & Temp. & Veloc. & Altitude \\
\hline Iguat-1 & $3,8(2-6)$ & $18(3-42)$ & $0,89(0,82-0,95)$ & $5,6(5,1-6,0)$ & $34,2(30-38)$ & $8,3(6,4-9,4)$ & $14,3(9-20)$ & $20,3(17,5-22)$ & $0,51(0,40-0,66)$ & 482 \\
\hline Iguat-2 & $4,3(3-7)$ & $5,8(4-8)$ & $0,95(0,92-0,98)$ & $6,0(5,5-6,5)$ & $24,2(20-27)$ & $7,5(6,2-9,3)$ & $151,8(16-538)$ & $21,5(20-23)$ & $0,40(0,30-0,50)$ & 400 \\
\hline Iguat-3 & $3,8(1-6)$ & $4,5(2-7)$ & $0,98(0,96-1,00)$ & $6,0(5,7-6,6)$ & $8,8(7-10)$ & $7,0(6,4-8,4)$ & $86(63-108)$ & $22,6(20,8-25)$ & $0,47(0,30-0,66)$ & 352 \\
\hline Iguat-4 & $0,7(0-1)$ & $0,7(0-1)$ & 0 & $6,2(5,3-7,2)$ & $9,3(7-13)$ & $7,6(6,8-9,0)$ & $112(61-180)$ & $20,7(18,2-23,9)$ & $0,44(0,24-0,66)$ & 295 \\
\hline Iguat-5 & $2(1-3)$ & $3,5(1-7)$ & $0,91(0,87-0,95)$ & $6,2(5,3-6,6)$ & $9,8(8-12)$ & $7,4(6,6-8,7)$ & $128,8(50-163)$ & $22,8(18,9-28)$ & $0,39(0,15-0,69)$ & 247 \\
\hline Iguat-6 & $4(1-6)$ & $6(3-8)$ & $0,92(0,86-0,98)$ & $6,0(5,3-6,4)$ & $9,3(8-12)$ & $7,6(6,6-9,3)$ & $122,3(62-170)$ & $21,4(19,2-24,2)$ & $0,44(0,17-0,66)$ & 235 \\
\hline Iguat-7 & $3,7(2-7)$ & $8,5(0-30)$ & $0,86(0,60-1,00)$ & $6,3(5,5-7,2)$ & $26,5(8-80)$ & $7,0(4,6-9,3)$ & $137,8(86-194)$ & $23,4(18,2-28,5)$ & $0,37(0,25-0,66)$ & 230 \\
\hline Iguat-8 & $5,0(3-8)$ & $10,3(6-16)$ & $0,87(0,77-0,94)$ & $6,9(6,4-7,4)$ & $9,7(8-11)$ & $7,5(6,5-8,6)$ & $123,3(97-165)$ & $21,3(18,3-24,7)$ & $0,36(0,25-0,50)$ & 226 \\
\hline
\end{tabular}



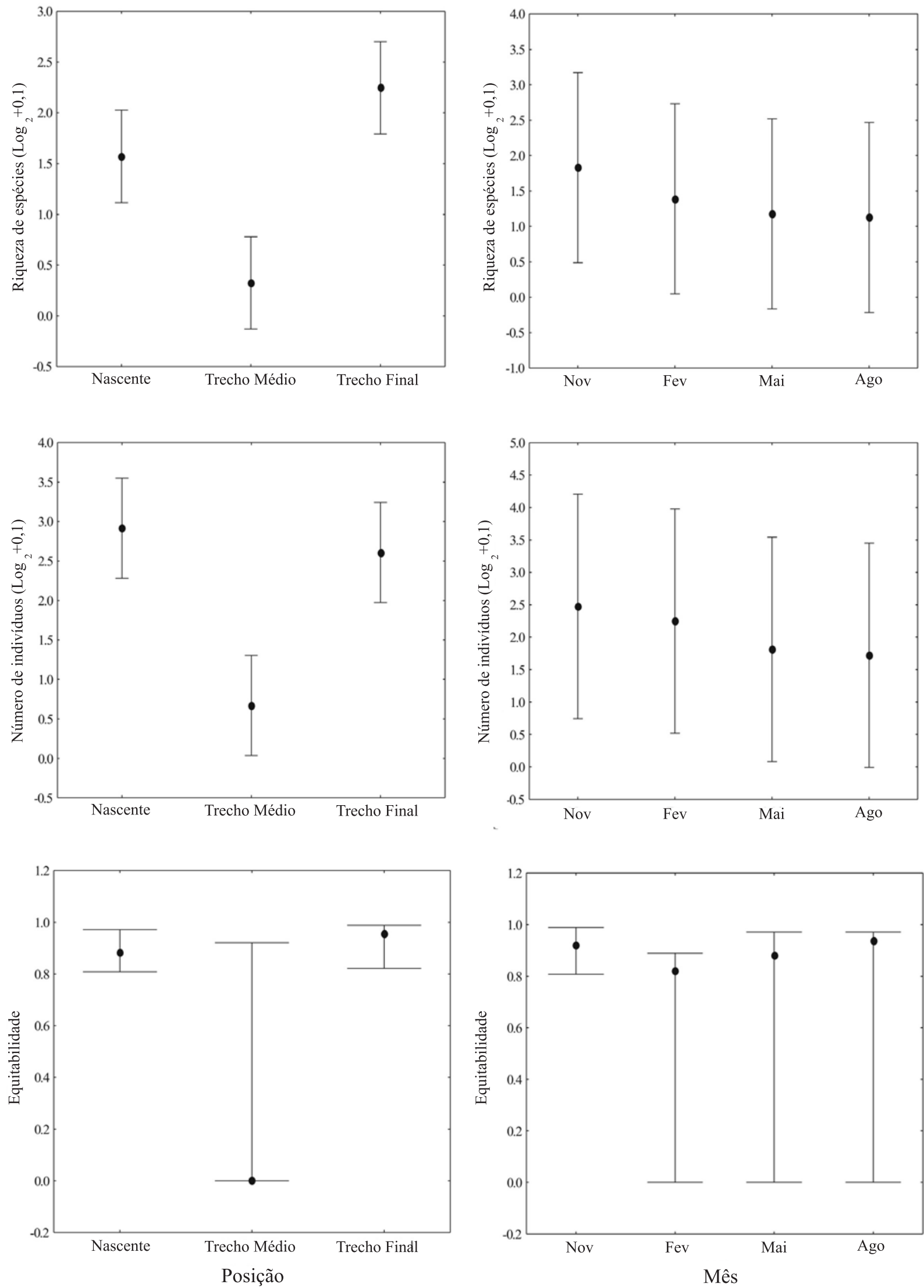

Fig. 2. Variação longitudinal e sazonal nas médias e intervalos de confiança para a riqueza e número de indivíduos e mediana e valores mínimos e máximos para a equitabilidade nas comunidades de peixes do rio Jogui, entre novembro/1999 e agosto/2000. 

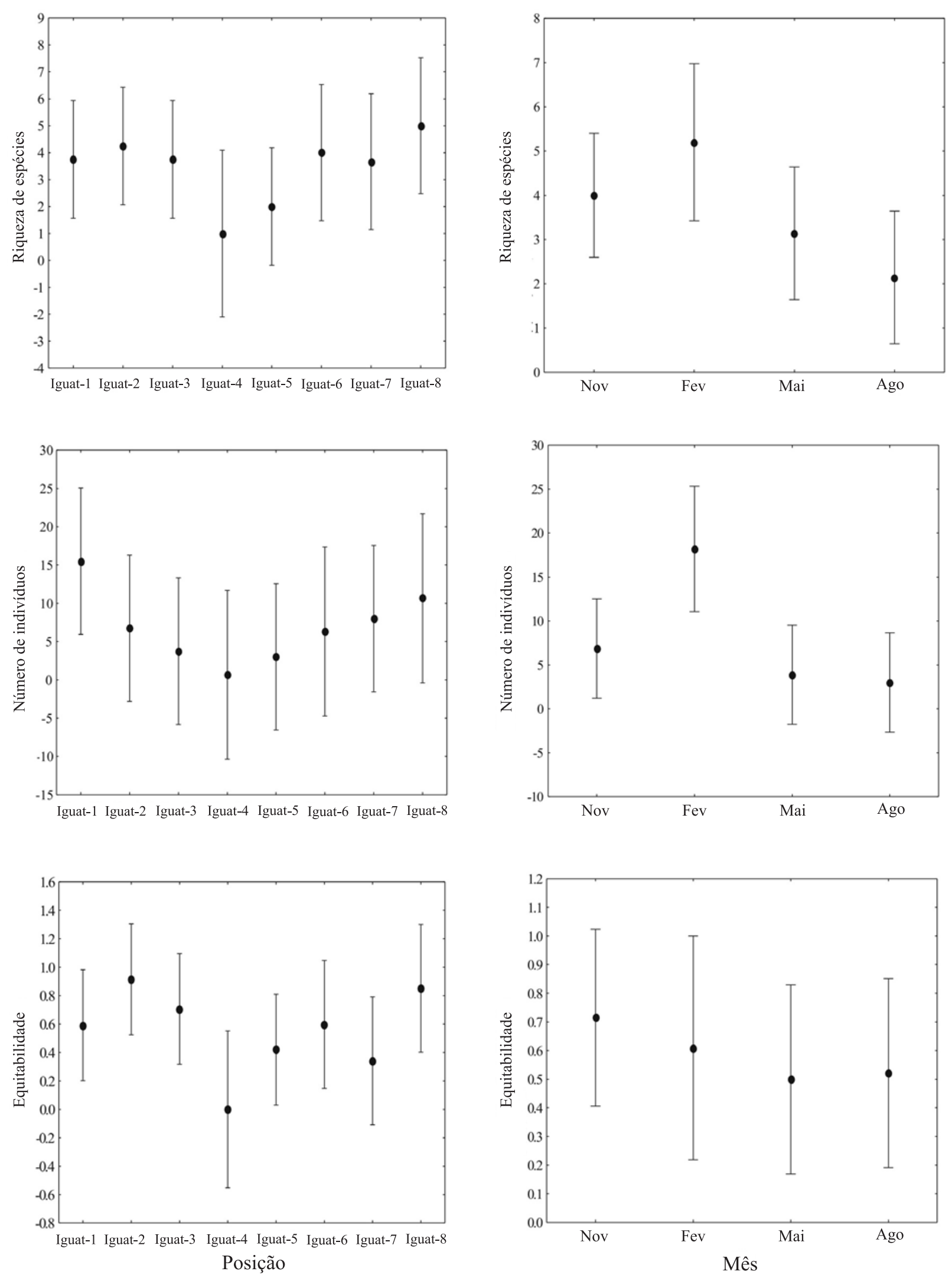

Fig. 3. Variação longitudinal e sazonal nas médias e intervalos de confiança para a riqueza, número de indivíduos e equitabilidade nas comunidades de peixes do rio Iguatemi, entre novembro/1999 e agosto/2000. 
Tabela II. Resultado da análise de variância da riqueza $\left(\log _{2}+0,1\right)$ e número de indivíduos $\left(\log _{2}+0,1\right)$ e Kruskall-Wallis da equitabilidade para as comunidades de peixes do rio Jogui, MS, entre novembro/1999 e agosto/2000.

\begin{tabular}{|c|c|c|c|c|c|c|}
\hline & \multicolumn{3}{|c|}{ Variação Longitudinal } & \multicolumn{3}{|c|}{ Variação Sazonal } \\
\hline & $\mathrm{F}$ & $\mathrm{P}$ & Poder & $\mathrm{F}$ & $\mathrm{P}$ & Poder \\
\hline Riqueza & 11,681 & 0,006 & 0,920 & 0,159 & 0,920 & 0,050 \\
\hline Num. indivíduos & 9,967 & 0,002 & 0,961 & 0,144 & 0,930 & 0,050 \\
\hline Graus de liberdade & & 2,7 & & & 3,6 & \\
\hline Kruskall-Wallis & $\mathrm{H}$ & $\mathrm{P}$ & Poder & $\mathrm{H}$ & $\mathrm{P}$ & Poder \\
\hline Equitabilidade & 2,209 & 0,104 & - & 1,916 & 0,059 & - \\
\hline
\end{tabular}

Tabela III. Resultado da análise de variância da riqueza, número de indivíduos e equitabilidade para as comunidades de peixes do rio Iguatemi, MS, entre novembro/1999 e agosto/2000.

\begin{tabular}{|c|c|c|c|c|c|c|}
\hline & \multicolumn{3}{|c|}{ Variação Longitudinal } & \multicolumn{3}{|c|}{ Variação Sazonal } \\
\hline & $\mathrm{F}$ & $\mathrm{P}$ & Poder & $\mathrm{F}$ & $\mathrm{P}$ & Poder \\
\hline Riqueza & 1,042 & 0,436 & 0,057 & 2,742 & 0,066 & 0,386 \\
\hline Num. indivíduos & 2,3 & 0,068 & 0,413 & 1,857 & 0,165 & 0,201 \\
\hline Equitabilidade & 0,589 & 0,734 & 0,050 & 0,257 & 0,855 & 0,050 \\
\hline Graus de liberdade & \multicolumn{3}{|c|}{7,21} & \multicolumn{3}{|c|}{3,25} \\
\hline
\end{tabular}

Tabela IV. Resultado da análise de regressão múltipla stepwise para os parâmetros das comunidades de peixes e as características selecionadas dos locais estudados (ns, não significativo; *, significativo a $5 \%$; **, significativo a $1 \%$ ).

\begin{tabular}{|c|c|c|c|}
\hline Rio & Var. Resposta & Var. Explanatória & Coefic. angular (b) \\
\hline & Riqueza $(\mathrm{gl}=5,6 ; \mathrm{n}=12)$ & $\mathrm{pH}$ & $0,832 \mathrm{~ns}$ \\
\hline & & Oxigênio Dissolvido & $0,413 *$ \\
\hline & & Temperatura & $-0,149 \mathrm{~ns}$ \\
\hline & & Turbidez & $0,032 * *$ \\
\hline & & Altitude Oxigênio & $0,010 *$ \\
\hline \multirow[t]{10}{*}{ Jogui } & Num. indiv. $(\mathrm{gl}=3,8 ; \mathrm{n}=12)$ & Oxigênio Dissolvido & $0,463 *$ \\
\hline & & Condutividade & $-0,102 \mathrm{~ns}$ \\
\hline & & Turbidez & $0,025 * *$ \\
\hline & & Altitude & $0,019 * *$ \\
\hline & Equitab. $\quad(\mathrm{gl}=2,9 ; \mathrm{n}=12)$ & Condutividade & $-0,068 *$ \\
\hline & & Altitude & $0,005 *$ \\
\hline & Riqueza $(\mathrm{gl}=4,24 ; \mathrm{n}=29)$ & Condutividade & $17,645 \mathrm{~ns}$ \\
\hline & & Velocidade & $-2,249 *$ \\
\hline & & $\mathrm{pH}$ & $0,485 \mathrm{~ns}$ \\
\hline & & Altitude & $0,003 \mathrm{~ns}$ \\
\hline \multirow[t]{5}{*}{ Iguatemi } & Num. indiv. $(\mathrm{gl}=2,26 \mathrm{n}=29)$ & Condutividade & $41,206 * *$ \\
\hline & & Oxigênio Dissolvido & $0,255 \mathrm{~ns}$ \\
\hline & Equitab. $(\mathrm{gl}=3,25 \mathrm{n}=29)$ & Velocidade & $1,455 \mathrm{~ns}$ \\
\hline & & $\mathrm{pH}$ & $-0,498 *$ \\
\hline & & Altitude & $-0,004 *$ \\
\hline
\end{tabular}

\section{DISCUSSÃO}

No rio Jogui, constatamos que não existe variação sazonal significativa para os descritores das comunidades analisados, mas existe variação longitudinal significativa para a riqueza e número de indivíduos coletados. No rio Iguatemi não constatamos variação significativa (longitudinal ou sazonal) para nenhum dos descritores das comunidades, contradizendo os pressupostos do conceito de rio contínuo (VANNOTE et al., 1980), bem como os resultados encontrados por ANGERMEIER \& KARR (1983); GARUtTI (1988); REYES-GavilÁn et al. (1996); Bistoni \& Hued (2002); Pavanelli \& Caramaschi (2003).
Dentre os efeitos das alterações longitudinais na estrutura das comunidades, o aumento da diversidade de espécies, em suas diferentes formas (riqueza, equitabilidade, entre outros) é um dos padrões mais evidentes, sendo constatada por inúmeros autores; então qual a explicação para a inexistência de um padrão claro de aumento da diversidade ao longo do rio Iguatemi?

Vários estudos têm constatado tanto adição quanto a substituição de espécies ao longo do gradiente lótico (Garutti, 1988; Angermeier \& Karr, 1983; Bistoni \& Hued, 2002), sendo que a importância de uma ou outra fonte de variação depende das características fisiográficas dos rios. 
O trecho final do rio Jogui apresenta velocidade média da água de $0,28 \mathrm{~m} / \mathrm{s}$, enquanto o trecho do rio Iguatemi próximo à foz do Jogui (Iguat-4 e Iguat-5) possuem velocidade média de $0,41 \mathrm{~m} / \mathrm{s}$. Desta maneira, é provável que a maior riqueza de espécies no trecho final do rio Jogui (25 espécies), quando comparado ao rio Iguatemi (8 espécies) nestes trechos, seja resultado da interação de dois fatores: 1) a migração de espécies entre os rios Iguatemi e Jogui, que procurariam este último devido à diferença de velocidade da água; 2) um maior fluxo de espécies entre o rio Jogui e sua planície de inundação, uma vez que a baixa velocidade não seria um fator limitante para estas espécies. A ocorrência de espécies no trecho final do rio Jogui, típicas de ambientes lênticos, corrobora esta hipótese (Hoplias malabaricus, Pyrhulina australis, Eigenmannia trilineata, Loricariichthys platymetopon, Hoplosternum littorale, Gymnotus sp. e Acestrorhynchus lacustris).

Analisando as associações entre espécies de peixes na bacia do rio Iguatemi, SúAREZ \& PETRERE-JúNIOR (2003) encontraram diferenças na composição de espécies entre os trechos superiores e inferiores da bacia; contudo, no presente trabalho constatamos que não existe diferença na diversidade de espécies ao longo do gradiente lótico. Este resultado corrobora a hipótese de STATZNER \& HigLER (1986) de zonação na distribuição das espécies, relacionada à existência de vários pequenos trechos de elevada correnteza, que podem estar determinando um padrão próprio de organização para cada trecho do rio.

A hipótese dos distúrbios intermediários (CONNELL, 1978) sugere que a diversidade de espécies possa ser aumentada por pequenos distúrbios que, atuando sobre a densidade populacional, permitem a ocorrência de maior número de espécies sem que a competição leve a exclusão de uma delas. A continuidade de um distúrbio por um longo período de tempo, no entanto, levaria algumas espécies a apresentarem baixas densidades populacionais, o que traz também o risco de extinção.

Os rios mais alterados na bacia do rio Iguatemi são o rio Puitã e a porção final do rio Jogui, onde praticamente inexiste vegetação ripária nativa e formam-se muitos bancos de areia no período da seca, fato que chegou a alterar parcialmente o leito original do rio Puitã em alguns trechos. Assim, o carreamento de sedimento para a porção final do rio Iguatemi, bem como as precárias condições de suas matas ripárias, estão levando a uma progressiva diminuição da diversidade de microhabitats, além da alteração das características hidrológicas originais.

BARRElla et al. (1994), comparando as comunidades de peixes dos rios Manso (MT) e Jacaré Pepira (SP), constataram maior diversidade ecomorfológica no rio Manso, atribuindo este resultado à maior disponibilidade de habitats, o que permite a ocorrência de maior número de espécies especialistas, quando comparado a um ambiente onde o desmatamento teria levado à predominância de espécies generalistas.

Esta hipótese, explicaria a baixa riqueza de espécies nos trechos finais do rio Iguatemi, uma vez que o nível de desmatamento e assoreamento em que se encontra a bacia vem a culminar no trecho final do rio, em sinergismo com o represamento do rio Paraná para a formação da represa de Itaipú (cerca de $20 \mathrm{~km}$ abaixo), alterando a densidade das espécies pré-existentes, e possivelmente levando à extinção pontual de algumas delas e à alteração no padrão de dominância.

No rio Jogui, a influência significativa da concentração de oxigênio, turbidez e altitude sobre a riqueza de espécies ressalta a diferença entre os trechos estudados, onde o trecho nascente se caracterizou pela maior concentração de oxigênio, enquanto o trecho final pela elevada turbidez, ambos com maiores valores de riqueza, quando comparados com o trecho médio (Tab. I). Esta maior riqueza encontrada nos trechos finais e iniciais do rio, levou à influência quase neutra da altitude sobre a riqueza de espécies.

Resultado similar pode ser observado para o rio Iguatemi, para a qual o conjunto de variáveis não manifestou influência significativa, mas a velocidade da água apresentou resultado da maior riqueza nos trechos finais, onde a velocidade da água foi menor.

A correlação positiva entre o número de indivíduos coletados e a condutividade no rio Iguatemi está relacionada à maior captura nos trechos iniciais do rio, fato também observado para a equitabilidade, que teve seus maiores valores em locais com maior velocidade da água (ainda que não significativa). Neste caso, ressaltase que apesar da altitude influenciar negativamente a equitabilidade, esta é praticamente neutra $(b=-0,004)$, sendo provavelmente um artifício estatístico de uma baixa estimativa de erro padrão do coeficiente angular b (erro padrão=0,001). Portanto, nossos resultados sugerem que a importância das variáveis limnológicas analisadas sobre as comunidades de peixes são principalmente resultado da diferenciação entre os trechos superiores e inferiores do rio.

Os rios Jogui e Iguatemi apresentam diferenças estruturais quanto à importância da variação longitudinal sobre a diversidade de espécies, com variação longitudinal significativa no rio Jogui e quase inexistente no rio Iguatemi, apesar de pertencerem à mesma bacia.

Não existem grandes variações geomorfológicas e limnológicas na bacia estudada, no entanto, a importância da condutividade na determinação da diversidade de espécies pode ser mero resultado da baixa riqueza observada nos trechos finais, uma vez que nos trechos iniciais, devido ao afloramento de rochas em ambos os rios, sempre eram encontrados maiores valores de condutividade.

Desta forma, concluímos que, para o rio Jogui, as variáveis ambientais que mais influenciam a riqueza e número de indivíduos coletados são aquelas associadas ao gradiente longitudinal, enquanto para o rio Iguatemi, não constatamos clara variação longitudinal ou sazonal, fato que pode ser atribuído à perda de integridade dos habitats, principalmente no trecho final da bacia, associado ao desmatamento e assoreamento na bacia.

Agradecimentos. À FAPESP (Processo 99/07719-1), Universidade Estadual de Mato Grosso do Sul, Universidade Estadual Paulista "Júlio de Mesquita Filho" e CNPq pelo financiamento parcial deste projeto. A K. G. Lopes, I. A. Aquino, M. L. Bezerra e C. L. Silva pela ajuda no trabalho de campo. A Flávio C. T. Lima (MZUSP), pelo auxílio taxonômico. A Antônio 
C. Beaumord, Walter Barrella, Francisco M. S. Braga, Roberto Goiten, Thomas M. Lewinsohn, Carlos E. C. Freitas e revisores anônimos pela leitura crítica e pelas sugestões.

\section{REFERÊNCIAS BIBLIOGRÁFICAS}

Angermeier, P. L. \& Karr, J. R. 1983. Fish communities along environmental gradients in a system of tropical streams. Environmental Biology of Fishes 9:117-135.

Barrella, W.; Beaumord, A. C. \& Petrere, M. 1994. Comparison between the fish communities of Manso River (MT) and Jacare Pepira River (SP), Brazil. Acta Biologica Venezuelica 12(2):11-20.

Bennemann, S. T.; Silva-Souza, A. T. \& Rocha, G. R. A. 1995 Composicion ictiofaunistica em cinco localidades de la cuenca del rio Tibagi, PR - Brasil. Interciência 20:1-13.

Bistoni, M. A. \& Hued, A. C. 2002. Patterns of fish species richness in rivers of the central region of Argentina. Brazilian Journal of Biology 62(4):753-764.

Connell, J. H. 1978. Diversity in tropical rain forest and coral reefs. Science 199:1302-1310.

Garutti, V. 1988. Distribuição longitudinal da ictiofauna em um córrego da região noroeste do Estado de São Paulo, Bacia do rio Paraná. Revista Brasileira de Biologia 48:747-759.

Gotelli, N. J. \& Entsminger, G. L. 2001. EcoSim: Null models software for ecology, version 7.0. S.I., Acquired Intelligence Inc. \& Kesey-Bear. Disponível em: <http:// homepages.together.net/ gentsmin/ecosim.htm>. Acesso em 10.12.2001

Jackson, D. A.; Peres-neto, P. R. \& Olden, J. D. 2001. What controls who is where is freshwater fish communities - the roles of biotic, abiotic and spatial factors. Canadian Journal of Fisheries and Aquatic Sciences 58:157-170.

Junk, W. J.; Bayley, P. B. \& Sparks, R. E. 1989. The Flood Pulse Concept in river-floodplain Systems. In: Dodge, D. P. ed Proceedings of the International Large River Symposium. Ottawa, p. 110-127. (Canadian special publication of fisheries and aquatic sciences, 106).

Loureau, M. \& Mouquet, N. 1999. Immigration and the maintenance of local species diversity. American Naturalist 154(4):427-440.

Macarthur, R. H. \& Levins, R. 1967. The limiting similarity, convergence, and divergence of coexisting species. American Naturalist 101:377-385.

Macarthur, R. H. \& Wilson, E. O. 1967. The theory of island biogeography. Princeton University Press, Princeton. 224p.

Pavanelli, C. S. \& Caramaschi, E. P. 2003. Temporal and spatial distribution of the ichthyofauna in two streams of the upper Rio Paraná basin. Brazilian Archives of Biology and Technology 46(2):271-280.

PiAnKA, E. R. 1966. Latitudinal gradients in species diversity: a review of concepts. American Naturalist 100:33-46.

Rathert, D.; White, D.; Sifneos, J. C. \& Hughes, R. M. 1999. Environmental correlates species richness for native freshwater fish in Oregon, USA. Journal of Biogeography 26:257-273.

Reash, R. J. \& PIGG, J. 1990. Physicochemical factors affecting the abundance and species richness of fishes in the Cimarron River. Proceedings of Oklahoma Academy of Sciences 70:23-28.

Reyes-Gavilán, F. G.; Garrido, R.; Nicieza, A. G.; Toledo, M. M. \& BRAÑA, F. 1996. Fish community variation along physical gradients in short streams of northern Spain and the disruptive effect of dams. Hydrobiologia 321:155-163.

Schoener, T. W. 1974. Resource partitioning in ecological communities. Science 185:27-39.

Statzner, B. \& Higler, B. 1986. Stream hydraulics as a major determinant of benthic invertebrate zonation patterns. Freshwater Biology 16:127-139.

Súarez, Y. R. \& Petrere-Júnior, M. 2003. Associações de espécies de peixes em ambientes lóticos da bacia do rio Iguatemi, Estado do Mato Grosso do Sul. Acta Scientiarum 25(2):361367.

Vannote, R. L.; Minshall, G. W.; Cummins, K. W.; Sedel, J. R. \& Cushing, C. E. 1980. The river continuum concept. Canadian Journal of fisheries and Aquatic Science 37:130-137.

Vila-Gispert, A.; García-Berthou, E. \& Moreno-Amich, R. 2002. Fish zonation in a Mediterranean stream: effects of human disturbances. Aquatic Sciences 64:163-170.

Recebido em agosto de 2004. Aceito em abril de 2006. ISSN 0073-4721

Artigo disponível em: www.scielo.br/isz 